Check for updates

Cite this: RSC Adv., 2019, 9, 1741

Received 25th December 2018 Accepted 8th January 2019

DOI: 10.1039/c8ra10547b

rsc.li/rsc-advances

\section{Knockdown of FOXO6 inhibits cell proliferation and ECM accumulation in glomerular mesangial cells cultured under high glucose condition}

\begin{abstract}
Yunqian Wang, $\dagger^{a}$ Lei Xue, $\dagger^{\mathrm{b}}$ Huicong Li, ${ }^{\mathrm{a}}$ Jun Shi ${ }^{* a}$ and Baoping Chen ${ }^{\mathrm{a}}$
Forkhead box O 6 (FOXO6), a FOX transcription factor, has been found to be involved in diabetes mellitus and related complications. However, the role of FOXO6 in diabetic nephropathy (DN) has not been fully understood. In the present study, we evaluated the functions of FOXO6 in high glucose (HG)-induced glomerular mesangial cells (MCs). The results showed that FOXO6 expression was significantly elevated in MCs after HG stimulation. Knockdown of FOXO6 by transfection with small interfering RNA (siRNA) targeting FOXO6 (siRNA-FOXO6) suppressed cell proliferation in MCs. The productions of extracellular matrix (ECM) components including collagen IV (Col IV) and fibronectin (FN) were markedly decreased after FOXO6 knockdown in MCs. Furthermore, knockdown of FOXO6 inhibited HG-induced activation of p38 MAPK signaling pathway in MCs. Collectively, these findings suggested that knockdown of FOXO6 inhibited cell proliferation and ECM accumulation in HG-induced MCs via inhibiting p38 MAPK signaling pathway. FOXO6 might be a beneficial therapeutic target for the prevention and treatment of DN.
\end{abstract}

\section{Introduction}

Since the incidence rate of diabetes mellitus is increasing worldwide, various diabetic complications have become major causes of morbidity and mortality in diabetic patients. ${ }^{1}$ Diabetic nephropathy (DN) is an important risk factor of renal dysfunction, which may lead to end-stage renal disease (ESRD) and life-threatening morbidity, resulting in heavy financial and health-care burdens globally. ${ }^{2,3}$ Therefore, controlling the incidence and morbidity of DN is an urgent biomedicine issue. Previous studies have demonstrated that hyperglycemia is a major contributing factor to the pathogenesis of $\mathrm{DN}^{.45}$ The phenomenon is attributed to the high glucose (HG)-caused excessive formation of extracellular matrix (ECM) along with expansion of glomerular mesangium, which are the hallmark pathological events of DN. ${ }^{6,7}$ Thus, inhibiting the ECM production and cell proliferation of glomerular mesangial cells can be an effective strategy for the prevention and treatment of DN.

Forkhead box (FOX) transcription factors are a protein family that consists of more than eighteen subfamilies. ${ }^{8}$ Proteins of the Fox family are evolutionarily conserved and play important roles in the regulation of diverse biological processes. ${ }^{9}$ There is

${ }^{a}$ Department of Nephrology, Huaihe Hospital of Henan University, No. 8 of Baobei Road, Kaifeng 475000, Henan Province, P. R. China. E-mail: shijun_hh@163.com; Tel: +86-0371-23906686

${ }^{b}$ Department of Endocrinology, Huaihe Hospital of Henan University, Kaifeng 475000, Henan Province, P. R. China

$\dagger$ These authors contributed equally to this work. convincing evidence that abnormal regulation of Fox genes is associated with developmental disorders and diseases, such as cancers, Parkinson's disease, and diabetes and diabetic complications. ${ }^{9,10}$ FOXO6, a noted member of Fox family, was reported to be involved in glucose metabolism in health and disease. ${ }^{11}$ Kim et al. ${ }^{11}$ demonstrated that FOXO6 activity is abnormally higher in the liver of mice with dietary obesity or type 2 diabetes (T2D). Furthermore, hepatic FOXO6 activity is induced by glucagon, while inhibited by insulin. FOXO6 integrates insulin signaling, contributing to the induction of hepatic gluconeogenesis, which is a life-sustaining process for maintaining blood glucose levels. ${ }^{12}$ However, the role of FOXO6 in DN remains unclear. In the present study, we aimed to investigate the functions of FOXO6 in HG-induced ECM production and cell proliferation of glomerular mesangial cells (MCs).

\section{Materials and methods}

\subsection{Cell culture and treatment}

Normal human MCs (Shanghai Academy of Life Sciences, Shanghai, China) were cultured in Dulbecco's modified Eagle's medium (DMEM; HyClone, Logan, UT, USA) supplemented with $10 \%$ fetal bovine serum (FBS; HyClone), $100 \mathrm{U} \mathrm{ml}^{-1}$ penicillin and $100 \mu \mathrm{g} \mathrm{ml}^{-1}$ streptomycin (HyClone). Cells were maintained in an incubator with $95 \%$ air and $5 \% \mathrm{CO}_{2}$ at $37{ }^{\circ} \mathrm{C}$. For some experiments, MCs were stimulated with $5.6 \mathrm{mM}$ glucose (NG) or $30 \mathrm{mM}$ glucose (HG) for different times. 
A

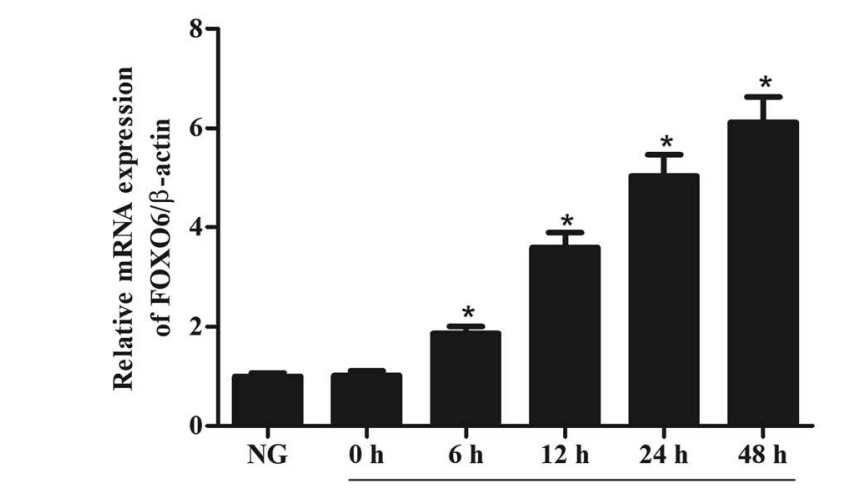

HG

B
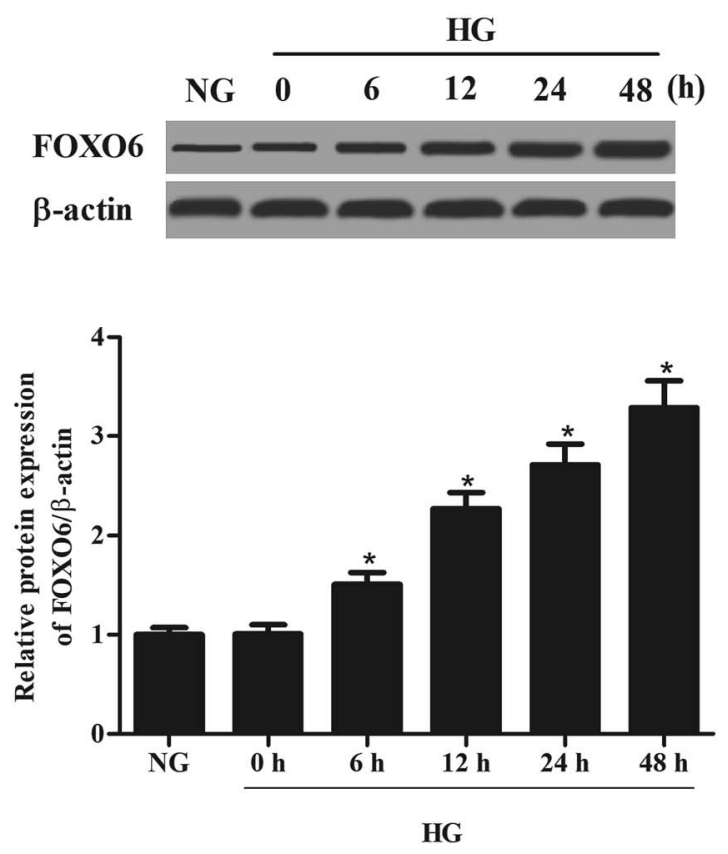

Fig. 1 Expression of FOXO6 in HG-stimulated MCs. Human MCs were treated with high glucose $(\mathrm{HG})$ for $0,6,12,24$ and $48 \mathrm{~h}$. The mRNA and protein levels of FOXO6 in MCs were respectively detected using qRTPCR (A) and western blot analysis (B). * $p<0.05$ vs. NG-stimulated MCs.

\subsection{Small interfering RNA (siRNA) FOXO6 and cell transfection}

The siRNA oligonucleotides including siRNA-FOXO6 and scrambled siRNA (siRNA-NC) were synthesized by Shanghai GenePharma (Shanghai, China). The transfection was conducted using opti-MEM and lipofectamine 2000 reagents (Invitrogen, Carlsbad, CA, USA) according to the manufacturer's instructions.

\subsection{Cell proliferation assay}

Cell proliferation of MCs was determined using MTT assay using a commercial kit (Beyotime Biotechnology, Shanghai, China) according to manufacturer's instructions. Briefly, siRNAFOXO6 or siRNA-NC was transfected into MCs and then
A

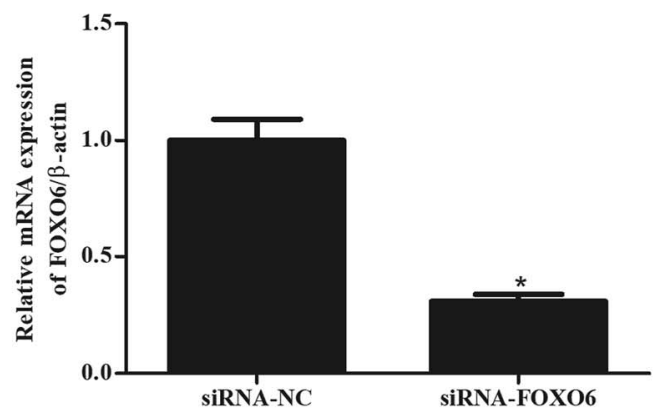

B
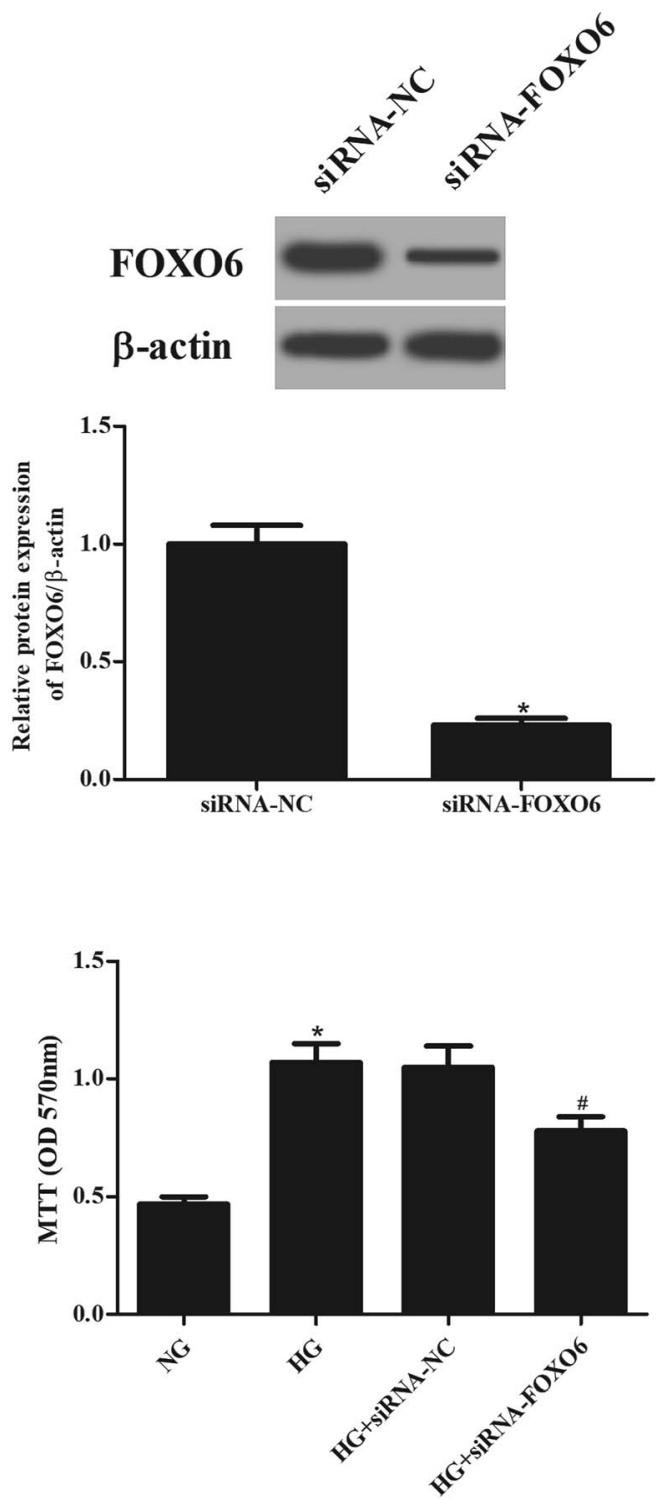

Fig. 2 Cell proliferation of HG-stimulated MCs was inhibited after knockdown of FOXO6. SiRNA-FOXO6 or siRNA-NC was transfected into MCs and then stimulated with $5.6 \mathrm{mM}$ glucose (NG) or $30 \mathrm{mM}$ glucose $(\mathrm{HG})$ for $48 \mathrm{~h}$. The mRNA and protein levels of FOXO6 in MCs were respectively detected using $\mathrm{qRT}-\mathrm{PCR}(\mathrm{A})$ and western blot analysis (B). (C) Cell proliferation was detected using MTT assay. * $p<$ 0.05 vs. NG-stimulated MCs; ${ }^{*} p<0.05$ vs. HG + siRNA-NC transfected MCs. 
A

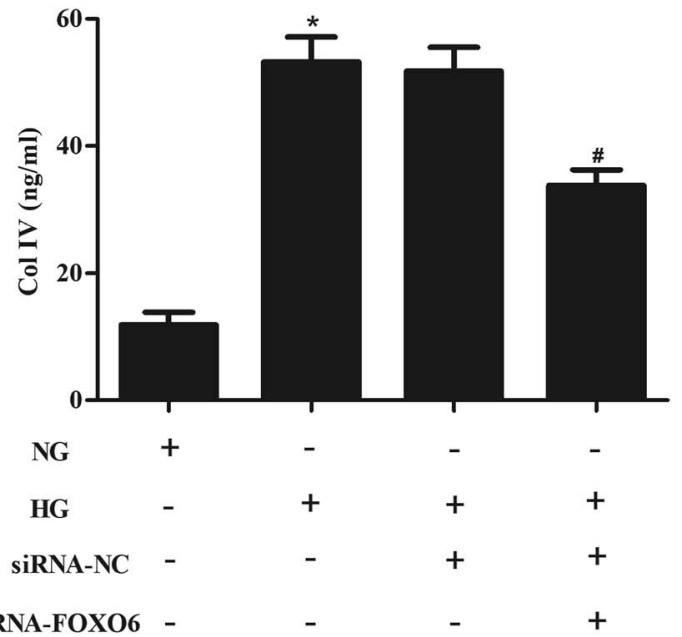

B

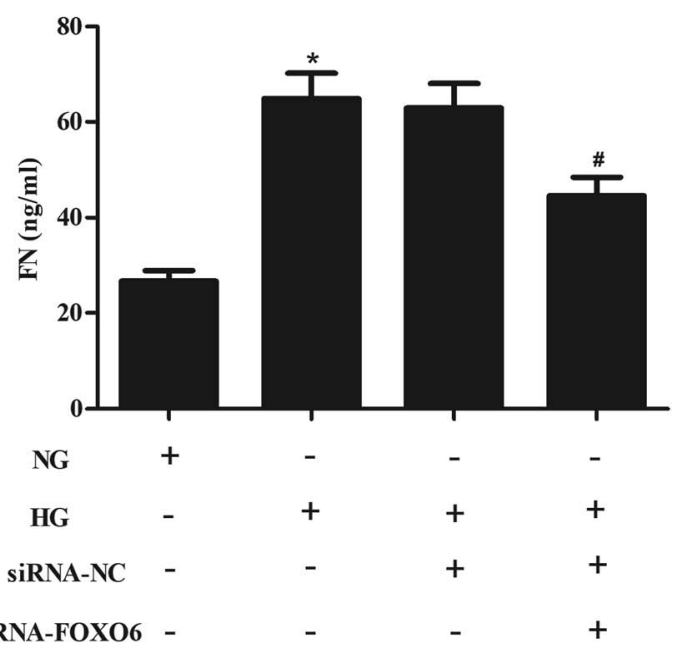

Fig. 3 Expression and secretion levels of FN and Col IV in HG-stimulated MCs were decreased after siRNA-FOXO6 transfection. Transfected MCs were treated with normal glucose (NG; $5.6 \mathrm{mM}$ ) or high glucose (HG; $30 \mathrm{mM}$ D-glucose) for $48 \mathrm{~h}$. (A and B) Secretion levels of Col IV and FN were measured using ELISA. $* p<0.05 \mathrm{vs}$. NG-stimulated MCs; ${ }^{\#} p<0.05$ vs. HG + siRNA-NC transfected MCs.

stimulated with $5.6 \mathrm{mM}$ glucose (NG) or $30 \mathrm{mM}$ glucose (HG) for $48 \mathrm{~h}$. Then, $20 \mu \mathrm{l}$ MTT stock solution at a concentration of $5 \mathrm{mg}$ $\mathrm{ml}^{-1}$ was then added to each well. After incubation for another $4 \mathrm{~h}$, dimethyl sulfoxide (DMSO) solution was added to dissolve the formazan crystals. After $5 \mathrm{~min}$, the absorbance was read using a microplate reader at $570 \mathrm{~nm}$.

\subsection{Quantitative real-time PCR (qRT-PCR)}

Total RNA was isolated from the cells using TRIzol reagent (Invitrogen). Then, $1 \mu \mathrm{g}$ RNA was utilized for the reverse transcription to synthesize cDNA using the SuperScript First-Stand Synthesis System (Invitrogen). After that, qRT-PCR was performed using Power SYBR Green PCR master mix (Applied Biosystem, Foster, CA, USA) on Real-Time PCR 7500 System
(Applied Biosystem) according to the manufacturer's instructions. Reaction conditions were listed as follows: initiated at $95{ }^{\circ} \mathrm{C}$ for $10 \mathrm{~min}$, followed by 45 cycles of denaturation at $95{ }^{\circ} \mathrm{C}$ for $15 \mathrm{~s}$, annealing at $57{ }^{\circ} \mathrm{C}$ for $15 \mathrm{~s}$, and hybridizationelongation at $72{ }^{\circ} \mathrm{C}$ for $30 \mathrm{~s}$. The primers were synthesized by Sangon Biotech (Shanghai, China): $\beta$-actin forward, $5^{\prime}$-CAT GTA CGT TGC TAT CCA GGC- $3^{\prime}$ and reverse, $5^{\prime}$-CTC CTT AAT GTC ACG CAC GAT-3'; FOXO6 forward, 5'-GGC CGC GCT CGT GTA CC- $3^{\prime}$ and reverse, $5^{\prime}$-TAC ACG AGC GCG GCC G-3'.

\subsection{Western blot analysis}

Cells were lysed in cell lysis buffer (Beyotime) and centrifuged at $12000 \mathrm{rpm}$ for $10 \mathrm{~min}$ at $4{ }^{\circ} \mathrm{C}$. Protein concentration was measured using Bradford method (Beyotime). Equivalent proteins $(50 \mu \mathrm{g})$ were separated on $10 \%$ SDS-PAGE and transferred to nitrocellulose membrane (Bio-Rad Laboratories, Hercules, CA, USA). Membranes were blocked with 5\% nonfat dry milk, followed by probing with anti-p38, anti-p-p38, and anti- $\beta$-actin (Santa Cruz Biotechnology, Santa Cruz, CA, USA) for overnight at $4{ }^{\circ} \mathrm{C}$. Then the membranes were incubated with secondary antibody conjugated with horseradish peroxidase (Santa Cruz). Protein bands were visualized using enhanced chemiluminescence reagent (Thermo Fisher Scientific, Waltham, MA, USA) and the signals were analyzed by Image J Software (National Institute of Health, Bethesda, MD, USA).

\subsection{ELISA}

The levels of collagen IV (Col IV) and fibronectin (FN) in cell culture supernatants were detected by commercial ELISA kits (R\&D Systems, Minneapolis, MN, USA) according to the manufacturer's instructions.

\subsection{Statistical analysis}

Results are expressed as mean \pm SEM. Statistical analysis of the data was performed using SPSS19.0 (SPSS Inc., Chicago, IL, USA). Student's $t$-tests or one way analysis of variance (ANOVA) with Tukey's multiple comparisons tests were performed to analyze the significance of differences between groups. A $p$ value less than 0.05 was considered to be significant.

\section{Results}

\subsection{The expression of FOXO6 is up-regulated in HG- stimulated MCs}

MCs were stimulated with $30 \mathrm{mM}$ glucose (HG) for $0,6,12,24$ and $48 \mathrm{~h}$. The mRNA and protein levels of FOXO6 were respectively detected using qRT-PCR and western blot analysis. As shown in Fig. 1A, mRNA level of FOXO6 was significantly upregulated in HG-stimulated $\mathrm{MCs}$ as compared to the NGstimulated MCs. It was also found that the protein level of FOXO6 was increased after HG stimulation (Fig. 1B). 


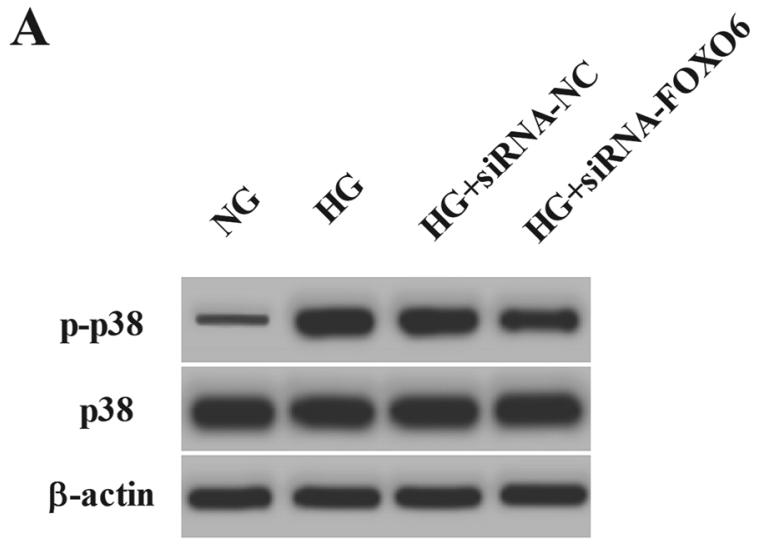

\section{B}

C

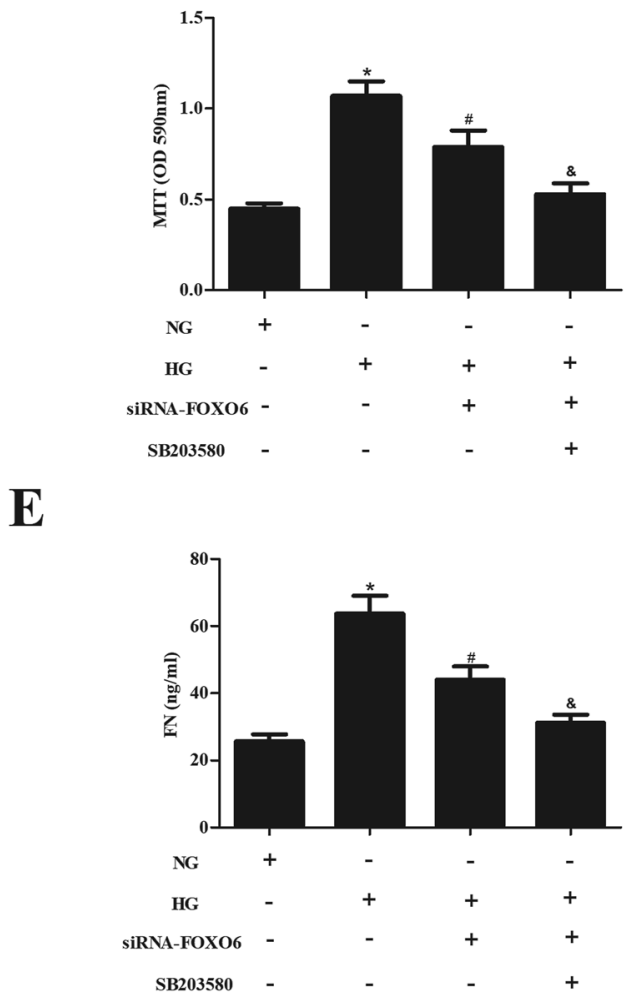

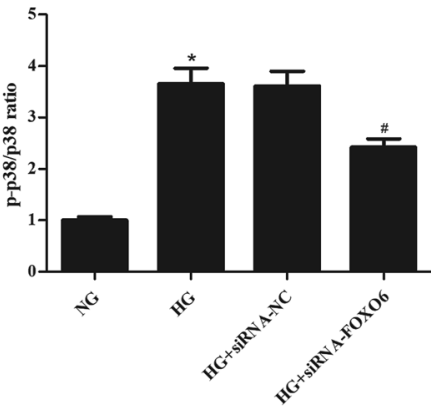

D

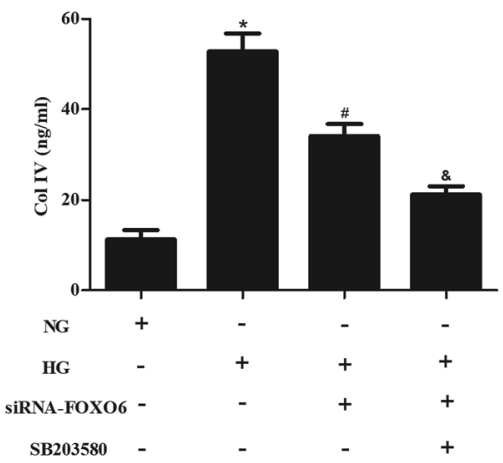

Fig. 4 Activation of p38 MAPK pathway in HG-treated MCs were blocked by FOXO6 knockdown. (A) Transfected MCs were treated with normal glucose (NG; $5.5 \mathrm{mM}$ ) or high glucose (HG; $30 \mathrm{mM}$-glucose) for $1 \mathrm{~h}$. The expressions of p38 and p-p38 were detected using western blot analysis. (B) Quantification of p-p38/p38. Transfected MCs were treated with normal glucose (NG; 5.6 mM) or high glucose (HG; 30 mM Dglucose) for $48 \mathrm{~h}$ in the presence or absence of a p38 inhibitor (SB203580). (C) Cell proliferation was detected using the MTT assay. (D and E) The production of Col IV and FN was detected by commercial ELISA kits. ${ }^{*} p<0.05$ vs. NG; ${ }^{*} p<0.05$ vs. HG; ${ }^{\xi} p<0.05$ vs. HG + siRNA-FOXO6.

\subsection{Knockdown of FOXO6 inhibits cell proliferation in MCs induced by HG}

To explore the role of FOXO6 in HG-stimulated MCs, siRNAFOXO6 or siRNA-NC was transfected into MCs. Results in Fig. 2A and B showed that FOXO6 expression was dramatically reduced after transfection with siRNA-FOXO6 in both mRNA and protein levels. HG stimulation significantly promoted cell proliferation of MCs, while knockdown of FOXO6 attenuated HG-induced cell proliferation (Fig. 2C).

\subsection{Knockdown of FOXO6 reduces the production of Col IV and FN in MCs induced by HG}

In order to evaluate the effect of FOXO6 knockdown on the ECM accumulation, the production of Col IV and FN was measured using ELISA. As shown in Fig. 3A and B, the levels of Col IV and FN were markedly increased in MCs stimulated by HG. However, knockdown of FOXO6 inhibited the HG-caused secretion of Col IV and FN. 


\subsection{Knockdown of FOXO6 inhibits the activation of p38 MAPK pathway in HG-treated MCs}

Previous studies have demonstrated that p38 MAPK pathway plays a crucial role in the HG-treated MCs. ${ }^{13,14}$ Therefore, we assessed the effect of FOXO6 knockdown on the activation of p38 MAPK pathway. Western blot analysis showed that HG stimulation resulted in significant increase in the expression of p-p38. However, the increased p-p38 expression was reduced after transfection with siRNA-FOXO6 (Fig. 4A and B). Moreover, SB203580 (p38MAPK pathway inhibitor) efficiently enhanced the inhibitory effects of siRNA-FOXO6 on cell proliferation, Col IV and FN production in HG-stimulated MCs (Fig. 4C-E).

\section{Discussion}

$\mathrm{DN}$ is a serious complication in diabetes patients with the major typical morphological changes in ECM accumulation. ${ }^{15}$ Due to increased amounts of ECM, basement membranes are thickened, the glomerular mesangial matrix and the tubulointerstitial space are expanded. ${ }^{\mathbf{1 5}}$ MCs are pericyte-like cells that make up the glomerulus mesangium. Pathological investigations have demonstrated that hyperglycaemia induced phenotypic changes in mesangial cells during DN. ${ }^{6}$ The synthesis of various ECM components and cell proliferation are significantly increased, contributing to the process of mesangium accumulation. ${ }^{6}$ There are copious evidences highlighted that inhibiting HG-induced ECM accumulation and MCs may contribute to preventing DN. Our previous study has demonstrated that silencing of progestin and adipoQ receptor 3 (PAQR3) suppresses ECM accumulation in HG-stimulated human MCs via PI3K/AKT signaling pathway. ${ }^{16}$ Scoparone, a natural compound, has been found to attenuate HG-induced ECM accumulation in rat MCs. ${ }^{17}$ These studies help us to understand the crucial roles of MCs in DN and suggest that therapeutic intervention for MCs in diabetic renal injuries can be an effective strategy for preventing $\mathrm{DN}$ in the future.

FOXO proteins are promising as central targets of insulin signaling and have been observed to be involved in various diabetic complications, such as diabetic retinopathy (DR) and DN. ${ }^{18,19}$ FOXO factors contribute to cell proliferation, apoptosis, response to oxidative stress, and regulation of metabolism. FOXO6 is an important member of the FOXO family that plays important roles in regulation of glucose metabolism. ${ }^{\mathbf{1 1}}$ FOXO6 is overexpressed in DR clinical vitreous samples and in HG-induced retinal pigment epithelium cells ARPE-19. Knockdown of FOXO6 inhibits HG-induced oxidative stress and apoptosis in ARPE-19 cells. $^{19}$ FOXO6 becomes deregulated in insulinresistant mice with obesity and type 2 diabetes. ${ }^{20}$ Diabetic $\mathrm{db} /$ $\mathrm{db}$ mice with selective FOXO6 knockdown in the insulinresistant liver display improved fasting glycemia with a concomitant reduction in fasting hyperinsulinemia. ${ }^{20}$ Genetically engineered mice with elevated FOXO6 activity in the liver augments gluconeogenesis, raising fasting blood glucose levels. While depletion of FOXO6 in liver suppresses gluconeogenesis, resulting in fasting hypoglycemia. ${ }^{12}$ However, the role of FOXO6 in MCs remains unclear. In the present study, we found that
FOXO6 expression is significantly up-regulated in HGstimulated MCs. Depletion of FOXO6 inhibits cell proliferation and ECM accumulation in MCs.

There is evidence that many pathways are involved in the progress of DN, such as Janus kinase (JAK)/Signal Transducer and Activator of Transcription protein (STAT), nuclear factor kappa B (NF- $\mathrm{B})$, Wnt, and MAPK signaling pathways. ${ }^{21-24}$ Previous study has reported that p38 MAPK signaling pathway is implicated in cell proliferation and ECM accumulation. Bao et $a l .{ }^{25}$ proved that activin A induces leiomyoma cell proliferation, ECM accumulation and myofibroblastic transformation of myometrial cells through activation of p38 MAPK signaling pathway. Morin, a natural bioflavonoid, inhibits cell proliferation and fibronectin accumulation in HG-stimulated rat MCs through suppressing the activation of p38 MAPK and JNK signaling pathways. ${ }^{14}$ Ishida et al. ${ }^{26}$ reported that stretchinduced overproduction of fibronectin in MCs is mediated by the activation of MAPK including extracellular signal-regulated kinase (ERK) and c-Jun NH2-terminal kinase (JNK). Protocatechuic acid (PCA) ameliorates HG-caused cell proliferation and ECM accumulation via inhibition of the p38 MAPK signaling pathway. ${ }^{\mathbf{1 3}}$ Therefore, we evaluated whether MAPK signaling pathway was involved in the effects of FOXO6. Our results showed that knockdown of FOXO6 inhibits HG-induced activation of p38 MAPK pathway in MCs.

\section{Conclusion}

In summary, the present study demonstrated that FOXO6 expression was significantly up-regulated in MCs after HG stimulation. Knockdown of FOXO6 in MCs suppressed cell proliferation and ECM accumulation. The effects of FOXO6 knockdown might be mediated by the inhibition of p38 MAPK signaling pathway.

\section{Conflicts of interest}

The authors declare no conflict of interest.

\section{Acknowledgements}

This study was supported by the Science and Technology Breakthrough Project of Henan Province Technology Committee, China (No. 152102310298).

\section{References}

1 J. M. Forbes and M. E. Cooper, Physiol. Rev., 2013, 93, 137188.

2 M. Kitada, K. Kanasaki and D. Koya, World J. Diabetes, 2014, 5, 342-356.

3 J. L. Gross, M. J. de Azevedo, S. P. Silveiro, L. H. Canani, M. L. Caramori and T. Zelmanovitz, Diabetes Care, 2005, 28, 164-176.

4 Y. S. Kanwar, L. Sun, P. Xie, F. Y. Liu and S. Chen, Annu. Rev. Phytopathol., 2011, 6, 395-423. 
5 F. P. Schena and L. Gesualdo, J. Am. Soc. Nephrol., 2005, 16, S30-S33.

6 C. W. Tung, Y. C. Hsu, Y. H. Shih, P. J. Chang and C. L. Lin, Nephrology, 2018, 23, 32-37.

7 R. M. Mason and N. A. Wahab, J. Am. Soc. Nephrol., 2003, 14, 1358-1373.

8 M. Katoh and M. Katoh, Int. J. Oncol., 2004, 25, 1495-1500.

9 O. J. Lehmann, J. C. Sowden, P. Carlsson, T. Jordan and S. S. Bhattacharya, Trends Genet., 2003, 19, 339-344.

10 M. L. Golson and K. H. Kaestner, Development, 2016, 143, 4558-4570.

11 D. H. Kim, T. Zhang, S. Lee and H. H. Dong, J. Diabetes, 2013, 5, 233-240.

12 D. H. Kim, G. Perdomo, T. Zhang, S. Slusher, S. Lee, B. E. Phillips, Y. Fan, N. Giannoukakis, R. Gramignoli, S. Strom, S. Ringquist and H. H. Dong, Diabetes, 2011, 60, 2763-2774.

13 Y. Ma, F. Chen, S. Yang, B. Chen and J. Shi, Biomed. Pharmacother., 2018, 98, 18-22.

14 Y. Q. Ke, C. Liu, J. B. Hao, L. Lu, N. N. Lu, Z. K. Wu, S. S. Zhu and X. L. Chen, Biomed. Pharmacother., 2016, 84, 622-627.

15 S. O. Kolset, F. P. Reinholt and T. Jenssen, J. Histochem. Cytochem., 2012, 60, 976-986.
16 H. Li, Y. Wang, B. Chen and J. Shi, Eur. J. Pharmacol., 2018, 832, 50-55.

17 Y. Wang, M. Wang, B. Chen and J. Shi, Eur. J. Pharmacol., 2017, 815, 376-380.

18 S. Lee and H. H. Dong, J. Endocrinol., 2017, 233, R67-R79.

19 Z. Zhou, J. Liu, C. Bi, L. Chen, Y. Jiao and L. Cui, J. Cell. Biochem., 2018, DOI: 10.1002/jcb.28252.

20 D. H. Kim, T. Zhang, S. Lee, V. Calabuig-Navarro, J. Yamauchi, A. Piccirillo, Y. Fan, R. Uppala, E. Goetzman and H. H. Dong, Endocrinology, 2014, 155, 1255-1267.

21 M. B. Marrero, A. K. Banes-Berceli, D. M. Stern and D. C. Eaton, Am. J. Physiol. Renal. Physiol., 2006, 290, F762F768.

22 A. Kuhad and K. Chopra, Life Sci., 2009, 84, 296-301.

23 M. Bose, S. Almas and S. Prabhakar, J. Invest. Med., 2017, 65, 1093-1101.

24 Y. Jiang, J. Liu, Z. Zhou, K. Liu and C. Liu, Exp. Clin. Endocrinol. Diabetes, 2018, DOI: 10.1055/a-0636-3883.

25 H. Bao, T. K. Sin and G. Zhang, Biochem. Biophys. Res. Commun., 2018, 504, 447-453.

26 T. Ishida, M. Haneda, S. Maeda, D. Koya and R. Kikkawa, Diabetes, 1999, 48, 595-602. 